Rev Esp Casos Clin Med Intern (RECCMI). 2020 (Ago); 5(2): 71-73

\title{
Rosai-Dorfman extranodal: una enfermedad rara con manifestación clínica común
}

\author{
María Carolina Fragozo-Ramos' ${ }^{1}$ Paola Andrea Ortiz-Marín', Marbel Karina Corzo-Pacheco², César Redondo-Bermúdez² \\ 'Servicio de Medicina Interna. ESE Hospital Universitario del Caribe. Universidad de Cartagena. Cartagena. Colombia \\ ${ }^{2}$ Servicio de Patología. ESE Hospital Universitario del Caribe. Universidad de Cartagena. Cartagena. Colombia
}

Recibido: 09/02/2020

Aceptado: 05/06/2020

En línea: 31/08/2020

Citar como: Fragozo-Ramos MC, Ortiz-Marín PA, Corzo-Pacheco MK, Redondo-Bermúdez C. Rosai-Dorfman extranodal: una enfermedad rara con manifestación clínica común. Rev Esp Casos Clin Med Intern (RECCMI). 2020 (Ago); 5(2): 71-73. doi: 10.32818/reccmi.a5n2a6.

Cite this as: Fragozo-Ramos MC, Ortiz-Marín PA, Corzo-Pacheco MK, Redondo-Bermúdez C. Extranodal Rosai-Dorfman: a rare disease with a common clinical manifestation. Rev Esp Casos Clin Med Intern (RECCMI). 2020 (Ago); 5(2): 71-73. doi: 10.32818/reccmi.a5n2a6.

Autor para correspondencia: María Carolina Fragozo-Ramos. carolinafragozo@gmail.com

\begin{aligned} Palabras clave \\ \hline$\triangleright$ Enfermedad \\ $\quad$ de Rosai-Dorfman \\ $\triangleright$ Hueso \\ $\triangleright$ Sistema nervioso central \\ $\triangleright$ Histiocitosis sinusoidal \\ $\triangleright$ Histiocitiosis de células \\ $\quad$ no-Langerhans \\ \hline\end{aligned}

\begin{tabular}{l}
\hline Keywords \\
\hline$\triangleright$ Rosai-Dorfman disease \\
$\triangleright$ Bone \\
$\triangleright$ Central nervous system \\
$\triangleright \quad$ Sinus histiocytosis \\
$\triangleright \quad$ Non-Langerhans cell \\
$\quad$ histiocytosis \\
\hline
\end{tabular}

\begin{abstract}
Resumen
La histiocitosis sinusoidal con linfadenopatía masiva o enfermedad de Rosai-Dorfman (ERD) es un trastorno proliferativo benigno de los histiocitos. Afecta con mayor frecuencia a niños y adultos jóvenes, pero puede ocurrir a cualquier edad. La enfermedad es más común en hombres e individuos de ascendencia africana. Es una entidad infrecuente con gran variedad de manifestaciones clínicas. Se presenta un caso de ERD extranodal de diagnóstico complejo con compromiso óseo y del sistema nervioso central. En este caso la biopsia fue concluyente, remarcando los aspectos distintivos de esta patología.
\end{abstract}

Abstract
Sinusoidal histiocytosis with massive lymphadenopathy or Rosai-Dorfman disease (RDD) is a benign proliferative
disorder of histiocytes. It is most frequently seen in children and young adults but can occur at any age. The disease
is more common in men and individuals of African descent. It is an uncommon entity with a variety of clinical
manifestations. We present a case of extranodal RDD of complex diagnosis with bone involvement and the central
nervous system. In this case, the biopsy was conclusive highlighting the distinctive aspects of this pathology.

\section{Puntos destacados}

$\triangleright$ La histiocitosis sinusoidal con linfadenopatía masiva o enfermedad de Rosai-Dorfman es una entidad con clínica común cuyo diagnóstico diferencial está entre las linfadenopatías y lesiones óseas.

$\triangleright$ La sospecha del clínico es imprescindible para orientar la toma de biopsia y decidir quiénes se benefician de un tratamiento específico.

\section{Introducción}

La enfermedad de Rosai-Dorfman (ERD) es una histiocitosis de células noLangerhans ${ }^{1,2}$ caracterizada por presencia de adenopatías cervicales acompañadas de síntomas constitucionales, velocidad de sedimentación globular (VSG) elevada, hipergammaglobulinemia policlonal, anemia moderada y trombocitopenia ${ }^{1,3}$. También presenta manifestación extranodal, siendo los órganos más afectados la piel, las órbitas, el tracto respiratorio superior y el hueso. El compromiso del sistema nervioso central (SNC) es usual en pa- cientes mayores y sin linfadenopatías'. Desde el punto de vista histológico, las lesiones presentan un infiltrado linfohistiocitario con la presencia intracitoplasmática de linfocitos intactos, fenómeno conocido como emperipolesis ${ }^{4}$. El tratamiento de la ERD se reserva para pacientes con compromiso sistémico o de órganos vitales ${ }^{5}$.

A continuación, se presenta un caso de ERD extranodal con compromiso óseo, del SNC y del tracto respiratorio superior.

\section{Caso clínico}

\section{Antecedentes}

Varón de 33 años de edad que acudía a Urgencias por 4 meses de cefalea intensa en hemicráneo izquierdo asociada a fiebre de bajo grado, pérdida involuntaria de peso, sudoración nocturna y edema progresivo 
Fragozo-Ramos MC, Ortiz-Marín PA, Corzo-Pacheco MK, Redondo-Bermúdez C. Rosai-Dorfman extranodal: una enfermedad rara con manifestación clínica común

facial izquierdo. Sin antecedentes patológicos destacables, ni hábitos tóxicos, ni exposiciones a otros agentes tóxicos o infecciosos. Negaba contacto con tuberculosis o antecedentes familiares de cáncer hematológico.

En la exploración a su ingreso: dolorido, hidratado, bien perfundido, con signos vitales normales, eupneico al aire ambiente. Presentaba edema descrito de predominio palpebral ipsilateral acompañado de dolor y sensibilidad a la palpación. No evidencia de linfadenopatía u organomegalia. En la evaluación neurológica: orientado, lenguaje fluido, presencia de parálisis completa facial central izquierda, con motilidad ocular normal, reflejo fotomotor y consensual conservados, sin signos meníngeos ni trastornos de la sensibilidad.

\section{Pruebas complementarias}

En la analítica sanguínea presentaba anemia moderada normocítica y normocrómica, conteo absoluto de leucocitos normal, diferencial con neutrofilia, proteína C reactiva (PCR) 9,4 mg/dl (valor normal hasta 0,30 mg/dl). La glucosa en sangre, las pruebas de función renal y hepática, y electrólitos fueron normales. La serología para hepatitis B, C y VIH fueron negativas.

La tomografía computarizada (TC) de cráneo sin contraste evidenció múltiples lesiones líticas en hueso frontal, esfenoidal y occipital (Figura 1).

La resonancia magnética (RM) craneal detectó un extenso proceso inflamatorio con compromiso óseo, de partes blandas, senos paranasales y mas toides, e imagen compatible con lesión isquémica focal en fase subaguda tardía capsulolenticular izquierda (Figura 2). Por hallazgos clínicos e imagenológicos, se consideró dentro de los diagnósticos iniciales: accidente cerebrovascular (ACV) isquémico gangliobasal izquierdo, pansinusitis crónica y osteomielitis crónica versus displasia fibrosa.

\section{Evolución}

Se inició esquema antibiótico con vancomicina, ceftriaxona y rifampicina, previa toma de hemocultivos, los cuales fueron negativos. Se decidió toma de biopsia más cultivo óseo por persistencia de síntomas. El examen histopatológico reveló un denso infiltrado celular inflamatorio mixto, rico en células plasmáticas e histiocitos, que mostraron emperipolesis (Figura 3). No se observó granuloma o necrosis, y en inmunohistoquímica las células histiocíticas fueron positivas para S-100 y CD68 con CD1a negativo (Figura 4), favoreciendo el diagnóstico de ERD extranodal. El paciente recibió pulsos de metilprednisolona, sin mejoría, y fue derivado a un centro oncológico para quimioterapia.

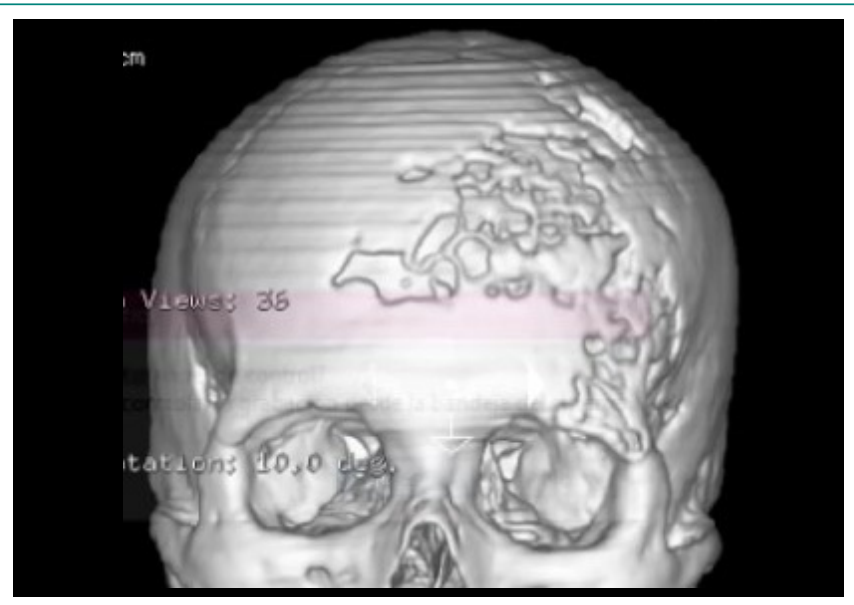

Figura 1. TC de cráneo con reconstrucción 3D. Compromiso de múltiples huesos

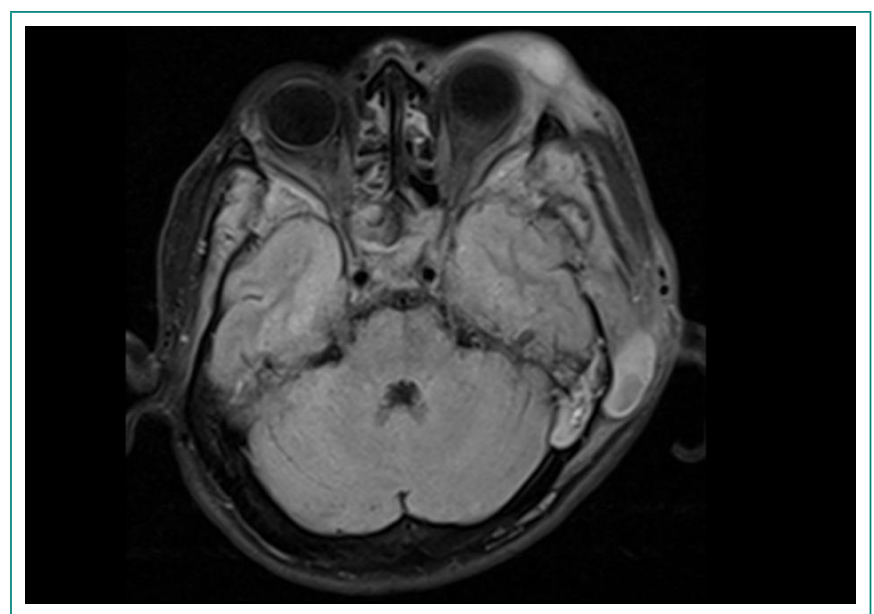

Figura 2. RM-T2 FLAIR, contrastada. Proceso inflamatorio en senos esfenoidales, celdillas etmoidales y mastoides con tumefacción de partes blandas extracraneales

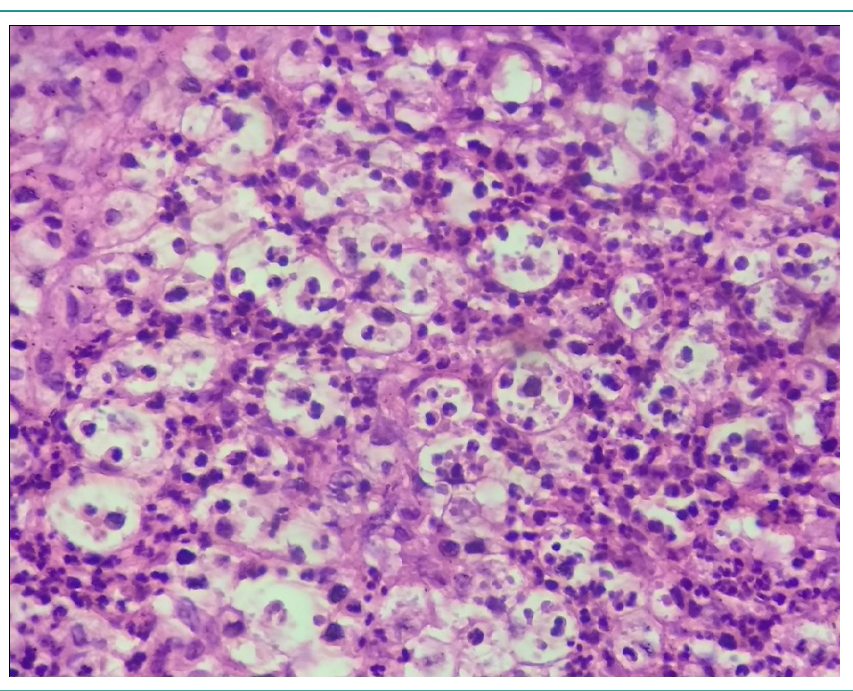

Figura 3. Hematoxilina y eosina, 40x. Proliferación de histiocitos con fenómeno de emperipolesis

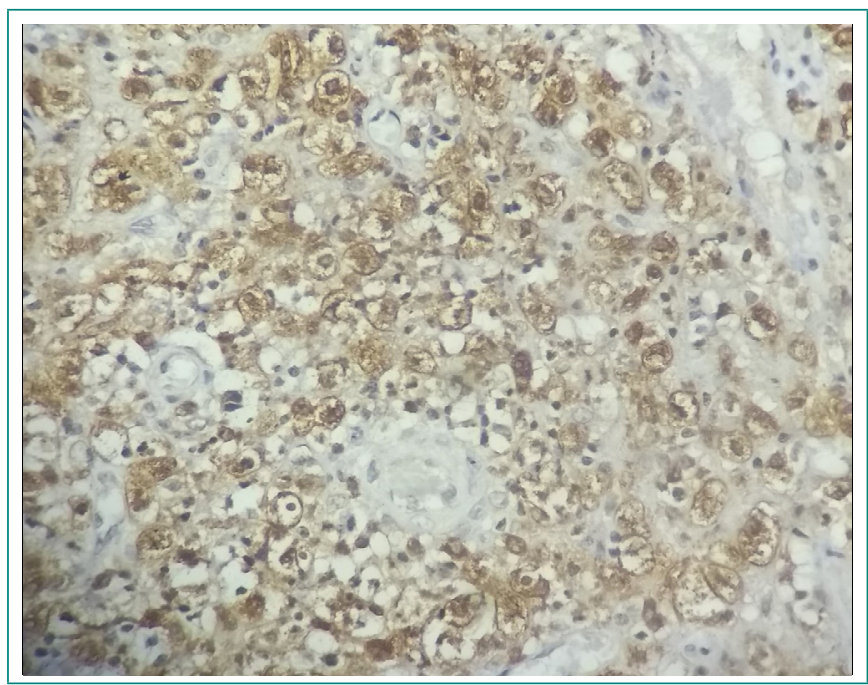

Figura 4. Técnica de inmunohistoquímica con S100, 40x. Histiocitos S100 positivos 


\section{Discusión}

La ERD se caracteriza por la acumulación de histiocitos activos ${ }^{1,4}$ y se considera una enfermedad rara (1/200.000)' de etiología desconocida. Puede ocurrir de forma aislada o en asociación con enfermedades autoinmunes, hereditarias o malignas ${ }^{1,6}$. También se ha relacionado con la infección por virus herpes, Epstein-Barr, citomegalovirus y VIH'1,5,6. Estudios recientes han encontrado mutaciones NRAS, KRAS, MAP2K' y ARAF, elevando la posibilidad de un origen clonal en algunas formas de la enfermedad, aunque en la mayoría de los pacientes no se identifica ningún tipo de mutación $n^{1,6}$

En la enfermedad clásica, destaca el compromiso nodal con linfadenopatías cervicales bilaterales, indoloras con o sin fiebre, sudores nocturnos y pérdida de peso. En el $43 \%$ de los pacientes puede presentarse como enfermedad extraganglionar con afectación multisistemica ${ }^{3}$. El compromiso de piel, cavidad nasal, senos paranasales y órbitas ocurre en el 11\% de los casos de ERD 1,3 . La afectación ósea ocurre en el 5-10\% de los casos, típicamente en asociación con enfermedad ganglionar, dolor óseo, lesiones líticas y fracturas patológicas, estas últimas poco comunes ${ }^{1,2}$. Las manifestaciones intratorácicas son descritas en el $2 \%$ de los pacientes e incluyen enfermedad pulmonar intersticial, nódulos pulmonares y derrames pleurales'. El 1\% de los casos cursa con expresión en tracto gastrointestinal, más frecuente en área ileocecal, apéndice y colon distal ${ }^{1,3}$. El compromiso cardíaco y renal ocurre en el 0,1-0,2\% y el $4 \%$ de los casos, respectivamente. En los riñones, la ERD puede debutar como una masa o infiltración difusa que genera hematuria, fallo renal y síndrome nefrótico' ${ }^{1}$.

El SNC se ve comprometido en el 5\% de los casos: el 75\% como lesiones intracraneales y el $25 \%$ como lesiones espinales. Los síntomas incluyen cefalea, convulsiones, trastornos de la marcha y alteraciones de pares craneales ${ }^{5}$ El aspecto radiográfico más común de la ERD intracraneal es una masa dural homogénea, solitaria extra axial que simula un meningiona ${ }^{1,5}$. Las lesiones espinales son más comunes en las regiones cervical y torácica y se presentan con mielopatía o síntomas de compresión medular 1,2,5. El estudio del líquido cefalorraquídeo muestra pleocitosis linfocítica, proteínas elevadas y glucosa baja'. Se recomienda realizar de forma rutinaria TC de cuello, tórax, abdomen y pelvis; en algunos casos, niveles de inmunoglobulinas cuantitativas, serología para VIH, hepatitis B y C, además de estudios de autoinmunidad según la historia clínica del paciente ${ }^{1,2}$

Se requiere la patología para realizar el diagnóstico definitivo. Las características histopatológicas remarcables incluyen la expansión sinusal de histiocitos grandes con citoplasma amplio, pálido con un gran núcleo hipocromático y nucléolo prominente'. La emperipolesis es típica pero no específica, pudiendo aparecer en otras enfermedades como linfoma de Hodgkin, mieloma múltiple, melanoma maligno, neuroblastoma y rabdomiosarcoma ${ }^{4}$ No obstante, la presencia de emperipolesis en ausencia de atipias con células que expresan proteína S100, pero son negativas para CD1a, confirma la ERD y ayuda a distinguirla de otras patologías similares?.

El tratamiento generalmente no se inicia hasta que el paciente esté sintomático o curse con progresión de la enfermedad³. La resección de la lesión puede ser curativa para la enfermedad unifocal y la reducción del volumen de la lesión puede justificarse en casos de obstrucción de la vía aérea superior, compresión de la médula espinal o lesiones grandes que causen compromiso del órgano afectado 1,3,8. En la enfermedad recurrente o refractaria puede ser necesario utilizar terapia sistémica o radiación. Reportes de casos han descrito terapia farmacológica exitosa incluyendo esteroides, metotrexato mercaptopurina, vincristina, rituximab, clofarabina, interferón alfa/interferón pegilado y cladribina ${ }^{3,6}$. Los esteroides corresponden a la terapia de primera línea y suelen ser útiles para reducir el tamaño de los ganglios y los síntomas ${ }^{1,6}$.
La quimioterapia podría considerarse para la enfermedad resistente a los esteroides o cuando éstos están contraindicados ${ }^{2}$. La radioterapia, que tiene eficacia principalmente en compromiso refractario en piel, órbita, enfermedad ósea y obstrucción de la vía aérea, también se ha implementado en pacientes cuyos síntomas persisten o recurren después de la resección de una enfermedad aislada y en aquellos pacientes que no son candidatos adecuados para cirugía y/o cuando otros tratamientos están contraindicados'.

El pronóstico depende del grado de compromiso. En general, los resultados son favorables especialmente para casos de enfermedad nodal y cutánea', 4 ; sin embargo, hasta un 12\% de los sujetos con ERD puede tener un desenlace mortal secundario a complicaciones sistémicas. El curso de la enfermedad es sustancialmente desfavorable en los casos de presentación multifocal con compromiso renal, hepático o de tracto respiratorio inferior?.

\section{Conclusiones}

Se presentó un caso de ERD extranodal. Pese a que es una enfermedad infrecuente, con signos y síntomas inespecíficos, se debe tener en cuenta dentro del diagnóstico diferencial de las linfadenopatías y las lesiones líticas óseas con compromiso del SNC en paciente joven. La sospecha clínica orienta la búsqueda y selección de quiénes se benefician de estudio histopatológico para su diagnóstico de certeza.

\section{Bibliografía}

1. Abla O, Jacobsen E, Picarsic J, Krenova Z, Jaffe R, Emile JF, et al. Consensus recommendations for the diagnosis and clinical management of Rosai-Dorfman-Destombes disease. Blood. 2018; 131 (26): 2877-2890. doi: 10.1182/blood-2018-03-839753.

2. Goyal G, Ravindran A, Young JR, Shah MV, Bennani NN, Patnaik MM, et al. Clinicopathological features, treatment approaches, and outcomes in Rosai-Dorfman disease. Haematologica. 2020 Jan 31; 105(2): 348-357. doi: 10.3324/haematol.2019.219626.

3. Namoglu EC, Hughes ME, Plastaras JP, Landsburg DJ, Maity A, Nasta SD. Management and outcomes of sinus histiocytosis with massive lymphadenopathy (Rosai Dorfman Disease). Leuk Lymphoma. 2019: 1-7. doi: 10.1080/10428194.2019.1703971.

4. Piris MA, Aguirregoicoa E, Montes-Moreno S, Celeiro-Muñoz C. Castleman disease and Rosai-Dorfman disease. Semin Diagn Pathol. 2018; 35(1): 4453. doi: 10.1053/j.semdp.2017.11.014.

5. Luo Z, Zhang Y, Zhao P, Lu H, Yang K, Zhang Y, et al. Characteristics of Rosai-Dorfman disease primarily involved in the central nervous system: 3 case reports and review of literature. World Neurosurg. 2017; 97: 58-63. doi: 10.1016/j.wneu.2016.09.084.

6. Triana-Pérez AB, Sánchez-Medina Y, Pérez-Del Rosario PA, Millán-Corada AM, Gómez-Perals LF, Domínguez-Báez JJ. Enfermedad de Rosai-Dorfman intracraneal. Presentación de un caso y revisión de la literatura. Neurocirugía (Astur). 2011; 22(3): 255-260. doi: 10.1016/S1130-1473(11)70021-5.

7. Mavrogenis AF, Igoumenou VG, Antoniadou $T$, Megaloikonomos $P D$, Agrogiannis G, Foukas P, et al. Rare diseases of bone: Erdheim-Chester and Rosai-Dorfman non-Langerhans cell histiocytoses. EFORT Open Rev. 2018; 3(6): 381-390. doi: 10.1302/2058-5241.3.170047.

8. Shah V, Mohyeldin A, London NR, Fritz J, Prevedello DM, Carrau RL, et al. When a meningioma isn't: endoscopic endonasal orbital decompression and biopsy of skull base Rosai-Dorfman disease treated previously with empiric radiation therapy. World Neurosurg. 2019 Dec 13; 135: 141-145. doi: 10.1016/j.wneu.2019.12.032. 